\title{
THE RESULTS OF TREATMENT IN KIENBÖGK'S DISEASE
}

\author{
Alford Dornan, Sheffield, England
}

Kienböck's disease is not common but it occurs sufficiently often to be of orthopa edic importance. The treatment and the prognosis are particularly important because most of the cases occur in heavy workers. For this reason it is of interest to review the results of treatment in a series of patients from an industrial area.

The condition was recognised by various observers before 1910, but it was Kienböck who first made a thorough study of the subject and tried to correlate the radiographic findings with the various clinical phases. He held that the condition was a malacia of the lunate bone from interference with the blood supply through the ligaments. This he attributed to injury which could be caused by a momentary subluxation of the bone ("jump subluxation"). Such fractures as might occur he regarded as secondary.

Persson (1945) agreed with the view originally advanced by Hultén (1928) that an important etiological factor is relative shortening of the lower end of the ulna with increased prominence of the articular surface of the radius, so that the lunate bone is exposed to greater stress in compression injuries of the wrist. This anatomical state, which Persson termed the " minus variation," was found in 8 per cent. of the general population, but in as many as ten of his nineteen cases of Kienböck's disease. This variation was present in a much smaller proportion of our cases. Persson advocated lengthening the ulna and reported good results. He believed that the operation relieved mechanical stress and so enabled the lunate bone to regenerate.

The condition has also been considered due to primary fractures, aseptic emboli and osteitis, but is now generally ascribed to avascular necrosis from traumatic interference with the blood supply.

TABLE I

Cases according to Age Groups

\begin{tabular}{|c|c|c|c|}
\hline Age in years & Cases & No operation & Operation \\
\hline $10-19$ & 5 & 3 & 2 \\
$20-29$ & 14 & 3 & 11 \\
$30-39$ & 13 & 11 & 2 \\
$40-49$ & 9 & 8 & 1 \\
50 and over & 2 & 2 & $16(37 \%)$ \\
\hline Total & 43 & $27(63 \%)$ & \\
\hline
\end{tabular}

For the purpose of this paper a review has been made of the patients with Kienböck's disease under my care in the past ten years. In all there were forty-six: three, treated conservatively, could not be traced; forty-two were seen and examined; the remaining patient failed to attend but the result was assessed from hospital records. Of the forty-three traced patients forty were male and three female; thirty-eight were heavy workers, including twenty-five coal-face workers, and five were light workers, including one housewife. The right wrist was affected in twenty-four cases and the left wrist in nineteen. Twenty-seven patients were treated conservatively. Of these, twenty-two were treated by immobilisation for an average period of three to four months; two were treated by rest and physiotherapy; and three had no specific treatment except a period of rest from work. Sixteen patients were treated by excision of the lunate bone, six as a primary measure and ten after a period of 
conservative treatment had failed to give relief. One patient was treated by arthrodesis of the wrist after an unsatisfactory result from excision.

Operation-The usual posterior approach was used, with a longitudinal incision, division of the sheath of extensor digitorum communis, and displacement of the tendons. Care was taken to make the excision complete, with the least possible injury to the other carpal bones. This was facilitated by continuous traction on the hand by an assistant. No post-operative immobilisation was used, movements of the fingers being encouraged from the beginning and wrist movements after a few days.

Results were classified as: excellent, no complaints and capable of full work without difficulty; good, capable of full work without loss of time, but symptoms after very heavy use; fair, improvement in symptoms but unable to resume heavy work; poor, no improvement, with persistent weakness and pain in the wrist.

It was found that the quality of the functional result was not always directly proportional to the final range of movement. Freedom from pain on movement, especially forced movement, and strength of grip were more important criteria of a good functional result. There was usually some weakness of grip, but in cases described as excellent this was slight.

Table II shows that of the patients who were traced 63 per cent. returned to full work after conservative treatment. In this group the three patients receiving no specific treatment had good or excellent results. Of the two treated by physiotherapy alone, one had a good and the other a fair result.

TABLE II

Analysis of Results of Conservative Treatment

\begin{tabular}{|c|c|c|c|c|c|}
\hline Age in years & Excellent & Good & Fair & Poor & Total \\
\hline $10-19$ & 0 & 3 & 0 & 0 & 3 \\
$20-29$ & 1 & 0 & 1 & 1 & 3 \\
$30-39$ & 5 & 2 & 3 & 1 & 11 \\
$40-49$ & 3 & 3 & 2 & 0 \\
50 and over & 0 & 0 & 1 & 1 & 2 \\
\hline Total & $9(33 \%)$ & $8(30 \%)$ & $7(26 \%)$ & $3(11 \%)$ & 27 \\
\hline
\end{tabular}

TABLE III

ANAlysis of Results of Excision

\begin{tabular}{|c|c|c|c|c|c|}
\hline $\begin{array}{c}\text { Years after } \\
\text { operation }\end{array}$ & Excellent & Good & Fair & Poor & Total \\
\hline $4-6$ & 3 & 1 & 2 & 1 & 7 \\
$2-4$ & 1 & 2 & 0 & 0 & 3 \\
$1-2$ & 2 & 0 & 2 & 0 & 4 \\
$0-1$ & 1 & 1 & 0 & 0 & 2 \\
\hline Total & $7(44 \%)$ & $4(25 \%)$ & $4(25 \%)$ & $1(6 \%)$ & 16 \\
\hline
\end{tabular}

Table III shows that 69 per cent. of patients returned to full work after excision of the lunate bone. One patient with a poor result was treated subsequently by arthrodesis of the wrist over two years ago; this gave considerable improvement, but he has not resumed his full pre-accident work as a steel worker. The result in the patient assessed from hospital notes is recorded as "fair" in the top line.

In attempting to compare the results of excision with those of conservative treatment it is difficult to select comparable groups because of the uncertain duration of the pathological process in a high percentage of cases, for instance those with obviously long-standing radiographic changes but with only a recent history of injury. Since excision of the lunate bone 
was not advised in elderly patients, or in the presence of gross arthritic changes, the choice of operation was virtually limited to patients under forty years of age. It was thought that the group of patients under the age of forty years who were treated conservatively was on the whole comparable with the group in which excision was undertaken. Reference to Table II shows that the results of conservative treatment in this age group differs little from the results in the whole series, 65 per cent. returning to full work.

\section{DISCUSSION}

This series of cases was drawn mostly from a mining community. The prognosis appears on the whole better than is usually supposed, because a fairly high proportion of patients returned to heavy work after either conservative or operative treatment. In fairly comparable groups the results of treatment whether by conservative measures or by removal of the lunate bone were not strikingly different.

In most patients treated conservatively with success the period of fixation was not long and only two patients required more than three months. Often the length of the history or the radiographic appearances showed that the condition had been latent for a long time and had been revealed only by a recent injury causing definite symptoms. In such cases a period of rest has been sufficient to restore quiescence and satisfactory function and to allow return to heavy work for a number of years. This seems to indicate a period of immobilisation for all patients, unless there are special reasons for primary excision of the lunate bone.

Arthrodesis of the wrist is valuable as a last resort, when there is persistent pain with gross osteoarthritis of the carpal joints. It does not, however, restore efficiency for many of the heavier types of work.

Has excision of the lunate bone a place in treatment? In those cases treated successfully by removal of the lunate bone, a striking result was the frequency of relief from aching pain. The strength of grip, although usually diminished just after the operation, gradually returned, though in some cases only after many months. None of the cases regarded as satisfactory has deteriorated. In my opinion removal of the lunate bone is justified in the few cases where there is no gross osteoarthritis, and where aching pain persists after efficient immobilisation for three or four months.

\section{REFERENCES}

Hultén, O. (1928): Acta radiologica 9, 155.

Kienвӧск, R. (1910): Fortschrifte auf dem Gebiete der Röntgenstrahlen, 16, 77. Persson, M. (1945): Acta chirurgica Scandinavica. Supplement 98, 51 and 79. 